\title{
Verbesserung der Kognition?
}

Fragestellung: Ist das synthetische Chaperon PBT2 bei der Behandlung der Huntington-Erkrankung verträglich und kann es die Kognition der Patienten verbessern?

Hintergrund: Die Aggregation von - mutanten - Proteinen wird zunehmend für die Initiation und Propagation von neurodegenerativen Erkrankungen verantwortlich gemacht. Im Tiermodell der Huntington-Erkrankung ist PBT2 wirksam bezüglich der Verbesserung motorischer Funktionen.

Patienten und Methodik: In der randomisierten doppelblinden placebokontrollierten Studie wurden 109 Patienten im frühen bis mittleren Stadium der Huntington-Erkrankung mit zwei Dosen von PBT2 oder Placebo über 26 Wochen behandelt. Primäre Endpunkte waren Sicherheit und Verträglichkeit des Medikaments. Sekundärer Endpunkt war die Kognition, wobei 13 individuelle Tests verwendet wurden.

Huntington Study Group Reach2HD Investigators. Safety, tolerability, and efficacy of PBT2 in Huntington's disease: a phase 2 , randomised, doubleblind, placebo-controlled trial. Lancet Neurol 2015; 14: $39-47$
Ergebnisse: PBT2 war in beiden Dosierungen sicher und verträglich. Nur einer der sekundären Endpunkte verbesserte sich statistisch signifikant in der höher dosierten Gruppe.

Schlussfolgerungen: PBT2 ist sicher und wird von Huntington-Patienten toleriert. Ein Effekt auf Exekutivfunktionen muss in einer größeren Studie belegt werden.

\section{Hier steht eine Anzeige.}

\section{- Kommentar von Albert C. Ludolph, Ulm}

\section{Exzellenter Ansatzpunkt}

Die Gemeinschaft der Huntington-Forscher ist in der Welt beispielhaft organisiert. Daher sind Studien möglich, die bei anderen, vergleichbaren Erkrankungen, kaum möglich wären. Diese Studie wurde in Australien und in den USA durchgeführt. Das Studienmedikament ist hinsichtlich des Wirkmechanismus hochaktuell, da es die Aggregation des mutanten Huntington-Proteins reduziert. Es ist nicht nur erfreulich, dass solche Studien durchgeführt werden können, sondern auch, dass solch innovative Konzepte mit Hilfe von nicht einer, sondern mehreren Dosen (hier zwei) angegangen werden. Es ist fast erstaunlich, dass bei einer solch kleinen Zahl von Patienten (insgesamt 109) ein Messinstrument (Trail Making Test Part B) einen statistisch signifikanten Effekt zeigte. Ich stimme den Autoren zu, dass dies ein exzellenter Ansatzpunkt ist, neue Studien durchzuführen; den gut organisierten Huntington-Forschern wird dies ohne weiteres möglich sein.

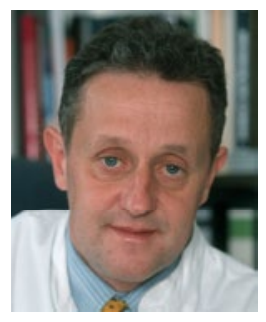

Prof. Dr. med. Albert C. Ludolph, Ulm

Ärztlicher Direktor, Klinik für Neurologie,

Universitätsklinikum Ulm

E-Mail: albert.ludolph@rku.de 